\title{
Angelicae Dahuricae Radix Inhibits Dust Mite Extract-Induced Atopic Dermatitis-Like Skin Lesions in NC/Nga Mice
}

\author{
Hoyoung Lee, Jun Kyoung Lee, Hyekyung Ha, Mee-Young Lee, \\ Chang-Seob Seo, and Hyeun Kyoo Shin
}

Herbal Medicine EBM Research Center, Korea Institute of Oriental Medicine, 483 Expo-ro, Daejeon Yuseong-gu, 305-811, Republic of Korea

Correspondence should be addressed to Hyeun Kyoo Shin, hkshin@kiom.re.kr

Received 8 July 2011; Revised 5 October 2011; Accepted 20 October 2011

Academic Editor: Yoshiyuki Kimura

Copyright (๑) 2012 Hoyoung Lee et al. This is an open access article distributed under the Creative Commons Attribution License, which permits unrestricted use, distribution, and reproduction in any medium, provided the original work is properly cited.

\begin{abstract}
We examined whether Angelicae Dahuricae Radix (AR) suppresses the development of atopic dermatitis (AD)-like skin lesions induced by Dermatophagoides farinae in NC/Nga mice. To investigate the effect of AR, we measured the AD severity score, measured plasma levels of IgE and histamine, and performed histological analysis in NC/Nga mice. We also confirmed the antiinflammatory effects of AR by measuring TARC/CCL17 production from LPS-treated RAW 264.7 cells and mRNA levels of TARC and MDC/CCL22 in TNF- $\alpha / \mathrm{IFN}-\gamma$-treated HaCaT cells. $10 \mathrm{mg}$ /day of AR extract was applied for 4 weeks to NC/Nga mice. Both the AR extract and $0.1 \%$ tacrolimus suppressed the development of AD-like skin lesions and reduced dermatitis scores of the back and ear skin. AR extracts caused an inhibition of histological changes induced by repeated application of $D$. farinae and a reduction of IgE and histamine levels in plasma $(P<0.05)$. Furthermore, NO production in LPS-treated RAW 264.7 cells was diminished in a dose-dependent manner, and hTARC production and TARC and MDC mRNA levels in TNF- $\alpha /$ IFN- $\gamma$-treated HaCaT cells were diminished by AR. The inhibitory effect of AR on NO, TARC and MDC production may be associated with the suppression of AD-like skin lesions in D. farinae-induced NC/Nga mice.
\end{abstract}

\section{Introduction}

Angelicae Dahuricae Radix (AR) is the dried root of Angelica dahurica ( $\mathrm{F}_{\mathrm{ISCH}}$. ) $\mathrm{B}_{\mathrm{ENTH}}$. et $\mathrm{H}_{\mathrm{OOKER}} \mathrm{F}$ (Umbelliferae). AR has been used for the treatment of colds, headaches, toothaches, coryza, and psoriasis in Korean traditional medicine [1]. There have been reports that AR has anti-inflammatory [2], antioxidant [3], and cytochrome P450 activity [4]. The major compounds of AR are imperatorin and coumarin. These compounds have also been shown to be effective agents for anticancer responses [5] as well as for the induction of betaendorphin [6], antihistamine [7], and antioxidative activity [8].

Atopic dermatitis (AD) is a chronic, relapsing skin disorder involving the interplay between migrating lymphocytes and epidermal keratinocytes [9]. AD is a clinical syndrome that is characterized by pruritic and dry skin lesions. Although topical steroids and immunosuppressive drug therapies are used to treat $\mathrm{AD}$ patients, these therapies result in adverse effects. At present, many researchers are exploring drugs traditionally used in herbal medicine, which have few adverse effects and excellent efficacy for dermatology [10]. Our study shows that AR is beneficial for treating AD-like skin lesions induced by the dust mite Dermatophagoides farinae on NC/Nga mice.

Th2 cells are induced to migrate to specific anatomic locations by thymus- and activation-regulated chemokines (TARC/CCL17) and macrophage-derived chemokines (MDC/CCL22), and regulated on activation which are specific ligands for CCR4 $[11,12]$. TARC and MDC have been found to be associated with $\mathrm{AD}$ in many reports. The serum levels of TARC and MDC have been shown to be markedly elevated and to correlate with disease severity in patients with $\mathrm{AD}[13,14]$. In the present study, we examined the effect of AR on D. farinae-induced AD-like skin lesions on NC/Nga mice. We also confirmed the anti-inflammatory effect of AR on LPS-induced RAW 264.7 cells and the antiallergy effect of AR on recombinant human tumor 
necrosis factor- $\alpha$ (TNF- $\alpha)$ /recombinant human interferon$\gamma(\mathrm{IFN}-\gamma)$-treated HaCaT cells.

\section{Materials and Methods}

2.1. Animals. Eight-week-old male NC/Nga mice were purchased from Central Laboratory Animal Inc. (Seoul, Korea) and housed in an air-conditioned room maintained at $24 \pm$ $2^{\circ} \mathrm{C}$ and $55 \pm 15 \%$ humidity. All procedures involving animals were conducted in accordance with the guidelines of the Institutional Animal Care and Use committee of the Korea Institute of Oriental Medicine (no. 09-173).

2.2. Chemicals and Reagents. Oxypeucedanin, oxypeucedanin hydrate, and byakangelicin were isolated from $A$. dahurica by a series of chromatography procedures. The purity of each compound was determined to be above 95\% by HPLC analysis. Nodakenin was obtained from Wako (Osaka, Japan). Imperatorin and isoimperatorin were purchased from ChromaDex (Santa Ana, CA, USA). HPLCgrade reagents, methanol, acetonitrile, and water were obtained from J.T. Baker (Phillipsburg, NJ, USA). Other chemicals were of analytical grade. A D. farinae ointment was purchased from Biostir Inc. (Hyogo, Japan). The positive control animals were treated with a $0.1 \%$ tacrolimus, an immunosuppressive drug (Protopic, Astellas, NY, USA). Human recombinant TNF- $\alpha$, IFN- $\gamma$, and TARC enzymelinked immunosorbent assay (ELISA) kits were obtained from R\&D Systems (MN, USA). Forskolin, $\mathrm{N}^{G}$-methylL-arginine (NMMA) and silymarin were purchased from Sigma-Aldrich (MO, USA). Mouse IgE and histamine ELISA kits are the products of Bethyl Laboratories Inc. (TX, USA) and Oxford biomedical research (MI, USA), respectively.

2.3. Preparation of AR Extract and Analysis of Chemical Components. Two-hundred grams of Angelicae Dahuricae Radix (AR; H-MAX, Korea) was obtained and extracted using a 10 -fold volume (w/v) of extraction solvent $(70 \%$ ethanol) to prepare the liquid extract. The extract was concentrated and then freeze dried to prepare a powdered extract $(62.19 \mathrm{~g})$. The extraction yield was $31.10 \%$.

HPLC was used to analyze the chemical components of the AR extract. The chemicals used to identify compounds in the AR extract included the following: oxypeucedanin, oxypeucedanin hydrate, byakangelicin, nodakenin, imperatorin, and isoimperatorin. The HPLC retention times of the six main coumarin compounds were approximately 8.9, 14.4, 14.9, 23.3, 28.3, and $31.1 \mathrm{~min}$ for nodakenin, oxypeucedanin hydrate, byakangelicin, oxypeucedanin, imperatorin, and isoimperatorin, respectively [15].

2.4. Sensitization. AD-like skin lesions were induced in 10week-old male NC/Nga mice using $D$. farinae extract as described by the manufacturer [16]. An ointment of $D$. farinae extract (Biostir AD) was purchased from Biostir Inc. (Hyogo, Japan) and applied twice per week for 4 weeks. Briefly, the hair on the upper back was shaved, and then $200 \mu \mathrm{L}$ of $10 \%$ (w/v) sodium dodecyl sulfate (SDS) was applied for barrier disruption to the shaved dorsal skin and both surfaces of each ear until the second treatment. After the second treatment, $200 \mu \mathrm{L}$ of $4 \%$ (w/v) SDS was applied to the skin and ears before topical application of $50 \mathrm{mg}$ of Biostir $\mathrm{AD}$. At the start of the experiment, mice were randomized to one of four groups ( $n=5$ each): untreated controls (70\% ethanol; Control), the Biostir AD-treated group (50 $\mathrm{mg} / \mathrm{mouse}$; . farinae), the Biostir AD with Protopictreated group (50 mg/mouse; tacrolimus), and the Biostir AD with AR extract-treated group $(10 \mathrm{mg} / \mathrm{mouse} / 70 \%$ ethanol; AR). AR extract was dissolved into $70 \%$ ethanol. Ethanol (70\%) was given to the animals in the vehicle control group.

2.5. Dermatitis Score. The severity of dermatitis was investigated macroscopically. The back skin and skin lesions on both ears were scored according to the following symptoms: erythema/hemorrhage, edema, excoriation/erosion, and scaling/dryness. The severity score was defined as the sum of individual scores, graded as follows: 0 (no symptom), 1 (mild), 2 (moderate), and 3 (severe).

2.6. Histopathology. After sacrificing the mice, the back skin and both ears were fixed in $10 \%(\mathrm{v} / \mathrm{v})$ neutral buffered formalin for $24 \mathrm{hr}$ at $4^{\circ} \mathrm{C}$. Tissues were next embedded in paraffin and thin sectioned ( $4 \mu \mathrm{m}$ thickness). Sections were then stained with $\mathrm{H} \& \mathrm{E}$ solution (hematoxylin and eosin, Sigma-Aldrich Inc., MO, USA) and subsequently mounted under cover slips using Dako-mounting medium (Dakocytomation, Denmark). Photographs were captured using a photometric Quanix digital camera, and images were assembled in Adobe Photoshop. To measure mast cell infiltration, skin sections were stained with toluidine blue, and the numbers of mast cells in four randomly chosen regions were counted.

2.7. ELISA. Blood samples were obtained from mice, and plasma was separated by centrifugation at $10,000 \mathrm{rpm}$ for $10 \mathrm{~min}$ at $4^{\circ} \mathrm{C}$. Plasma was collected and stored at $-80^{\circ} \mathrm{C}$. Total plasma IgE and histamine levels from treated NC/Nga mice were measured by ELISA according to the manufacturer's instructions.

2.8. Cell Culture. The murine macrophage cell line RAW 264.7 was obtained from the American Type Culture Collection (ATCC, Rockville, MD, USA). The cells were maintained in DMEM media containing 5.5\% FBS and 1\% $\mathrm{P} \& \mathrm{~S}(100 \mathrm{U} / \mathrm{mL}$ of penicillin and $100 \mu \mathrm{g} / \mathrm{mL}$ of streptomycin) in a humidified $5 \% \mathrm{CO}_{2}$ atmosphere. The human keratinocyte cell line $\mathrm{HaCaT}$, provided by professor Nakyung Lee at Sejeong University, was cultured in DMEM with $10 \%$ (v/v) FBS and 1\% P\&S. RAW 264.7 cells and HaCaT cells were seeded at a density of $2.5 \times 10^{3}$ cells/well and $1.0 \times$ $10^{3}$ cells/well, respectively, for proliferation analysis for $24 \mathrm{hr}$. The cell counting Kit-8 reagent (CCK-8, Dojindo, Japan) was added and incubated for $4 \mathrm{hr}$. We measured the absorbance at $450 \mathrm{~nm}$ using a Benchmark Plus ELISA reader (BIO$\mathrm{RAD}$, Japan), and the percentages of proliferating cells were calculated. 


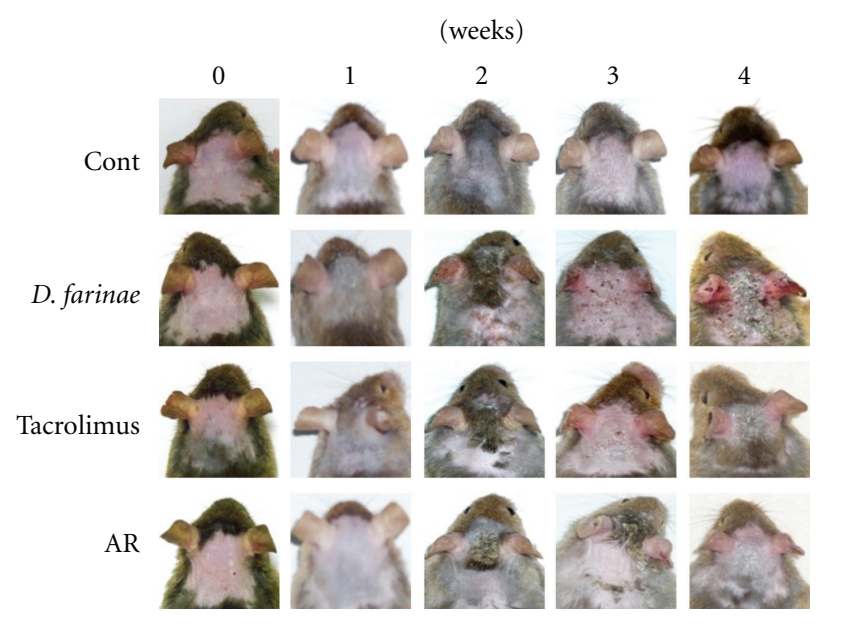

(a)

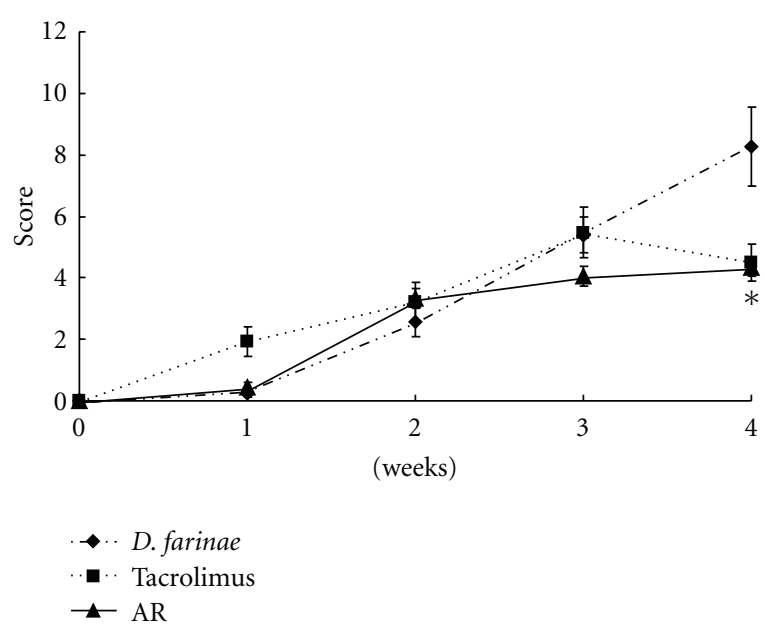

(b)

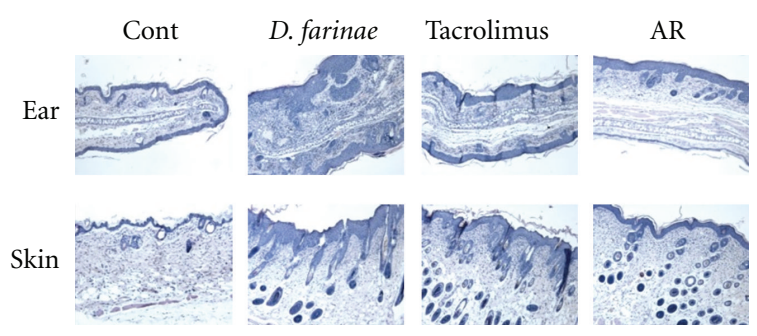

(c)

FIGURE 1: Severity scores and histological changes of treated backs and ears in NC/Nga mice. (a) Representative features and histological changes following consecutive administration of Angelicae Dahuricae Radix (AR) or tacrolimus to Dermatophagoides farinae-induced ADlike lesions on the back and ears. The images show the back and ears 4 weeks after sensitization. (b) Effect of consecutive administration of AR or tacrolimus on dermatitis score of $D$. farinae-induced AD-like lesions on the back and ears. AR or tacrolimus were administrated once daily for 4 weeks. (c) Histological features of AD-like lesions treated with AR. The dermatitis scores were evaluated by the procedures described in Materials and Methods (mean $\pm \operatorname{SEM}(n=5),{ }^{*}: P<0.05$, compared with $D$. farinae-induced group).

2.9. Measurement of Nitric Oxide (NO). RAW 264.7 cells were seeded at a density of $2.5 \times 10^{5}$ cells in a 48 -well plate for the NO assay. After 18 hour incubation, the cells were stimulated with $1 \mu \mathrm{g} / \mathrm{mL}$ of LPS in the presence or absence of AR extracts for the indicated periods. A griess reagent system (Promega. WI. USA) was used to measure the production and inhibition of NO in culture supernatants. Briefly, a 50$\mu \mathrm{L} /$ well sample was incubated at room temperature with $1 \%$ sulfanilamide for $10 \mathrm{~min}$ and $1 \% \alpha$-naphthylamine for $10 \mathrm{~min}$. The absorbance was evaluated at $535 \mathrm{~nm}$ using a calibration curve with standard.

2.10. Measurement of hTARC. HaCaT cells were seeded at a density of $1.0 \times 10^{6}$ cells/well in 6-well plates for the hTARC assay. After $24 \mathrm{hr}$, the cells were washed and incubated with $1 \mathrm{~mL}$ serum-free media containing TNF- $\alpha(10 \mathrm{ng} / \mathrm{mL}) / \mathrm{IFN}-\gamma$ $(10 \mathrm{ng} / \mathrm{mL}$ ) plus AR extract for $24 \mathrm{hr}$. Cells were subsequently incubated with AR extract $(200,500$, or $1000 \mu \mathrm{g} / \mathrm{mL})$ for $24 \mathrm{hr}$; other groups of cells were treated with forskolin (10 and $30 \mu \mathrm{M})$ and silymarin (20 and $50 \mu \mathrm{g} / \mathrm{mL}$ ) as positive controls. The absorbance was evaluated using a Benchmark
Plus ELISA reader at $450 \mathrm{~nm}$ using a calibration curve with a standard.

2.11. Gene Expression (RT-PCR). After a 24-hr incubation of cells as described above, total RNA was isolated using TRIzol reagent (Invitrogen Life Technologies, CA, USA). cDNA was synthesized from total RNA $(2 \mu \mathrm{g})$ using an iScript Select cDNA Synthesis kit (Bio-Rad, CA, USA) according to the manufacturer's instructions. For PCR amplification, we used the following specific primers for the mRNAs: TARC (forward: $5^{\prime}$-actgctccagggatgccatcgttttt- $3^{\prime}$, reverse: $5^{\prime}$-acaaggggatgggatctccetcactg- $3^{\prime}$ ); MDC (forward: $5^{\prime}$-aggacagagcatggctcgcctacaga- $3^{\prime}$, reverse: $5^{\prime}$-taatggcagggaggtagggctcctga$3^{\prime}$ ); hGAPDH (forward: $5^{\prime}$-aagggtcatcatctctgccc- $3^{\prime}$, reverse: $5^{\prime}$-gtgatggcatggactgtggt- $\left.3^{\prime}\right)$, which were expected to yield fragments of $270 \mathrm{bp}, 362 \mathrm{bp}$, and $204 \mathrm{bp}$, respectively.

2.12. Statistical Analysis. Data are reported as the means \pm SEM and were compared by ANOVA followed by the Bonferroni multiple comparison method. For score data analysis, data were compared by Wilcoxon statistics 
after Kruskalis-Wallis test (SYSTAT 10.0., SPSS Inc., USA) A $P$-value $<0.05$ was defined as statistically significant.

\section{Results}

3.1. Histological Analysis. Skin from treated NC/Nga mice was investigated once a week for 4 weeks (Figure 1(a)). The control group showed no physical signs of dermatitis, erythema/hemorrhage, edema, excoriation/erosion, or scaling/dryness. In contrast, the severity scores of the $D$. farinae group gradually increased after sensitization. The groups receiving tacrolimus and AR treatments both showed a reduction in the development of $\mathrm{AD}$-like skin lesions, and a significant difference in lesions was observed between 2 and 4 weeks. The severity scores are shown in Figure 1(b). We show that the tacrolimus and AR treatments suppressed ADlike skin lesions compared with treatments with $D$. farinae alone.

3.2. Histopathological Observation. Histopathological findings from the back skin and ear skin are shown in Figure 1(c). Topical application of $D$. farinae increased the thickness of back and ear skin. In the tacrolimus- and AR-treated groups, there were fewer prominent inflammatory changes, such as erosion and hyperplasia of epidermal and dermal tissues.

3.3. Plasma Levels of Histamine and IgE. The histamine level in plasma is shown in Figure 2(a). The D. farinae-treated group $(1.27 \pm 0.15 \mu \mathrm{M})$ had an increase in histamine levels compared with the control group $(0.91 \pm 0.16 \mu \mathrm{M})(P<$ $0.01)$. AR treatment reduced the histamine level significantly $(0.82 \pm 0.0 .08 \mu \mathrm{M} ; P<0.05)$. The group receiving tacrolimus also had decreased histamine levels $(1.03 \pm 0.10 \mu \mathrm{M})$, but this decrease did not reach statistical significance.

Similar trends were seen with IgE levels in plasma. The control group secreted $56.67 \pm 14.91 \mathrm{ng} / \mathrm{mL}$ IgE. However, the $D$. farinae-treated group had a 4.7 -fold increase in $\operatorname{IgE}$ plasma level $(266.54 \pm 14.92 \mathrm{ng} / \mathrm{mL})$. When AR was applied to the AD-like lesions, the IgE level was reduced 1.44-fold $(184.46 \pm 19.70 \mathrm{ng} / \mathrm{mL})$. However, there was no significant difference in IgE levels in the tacrolimus group compared with $D$. farinae-treated group (Figure 2(b)).

3.4. Effects of AR on NO and hTARC Production. The effects of AR on NO production were examined in LPS-induced RAW 264.7 cells. Control cells released $2.21 \pm 0.18 \mu \mathrm{M}$ of nitrite during $18 \mathrm{hr}$ of incubation. Upon exposure to LPS alone, the cells released 51.01 $\pm 2.28 \mu \mathrm{M}$ nitrite (Figure 3(a)). For the positive control, NMMA inhibited LPS-induced nitrite production in a dose-dependent manner, corresponding to a $20 \%$ inhibition at $10 \mu \mathrm{M}$ and a $56 \%$ inhibition at $100 \mu \mathrm{M}$. Additionally, NO production was inhibited by $\mathrm{AR}$ in a dose-dependent manner, corresponding to an $8 \%$ inhibition at $15 \mu \mathrm{g} / \mathrm{mL}$ and a $51 \%$ inhibition at $150 \mu \mathrm{g} / \mathrm{mL}$.

The effects of AR on hTARC production were examined in TNF- $\alpha / \mathrm{IFN}-\gamma$-induced HaCaT cells. As a positive control for decreasing hTARC levels, we used silymarin (20, $50 \mu \mathrm{g} / \mathrm{mL})$ and forskolin $(10,30 \mu \mathrm{M})$ to inhibit cAMP. The

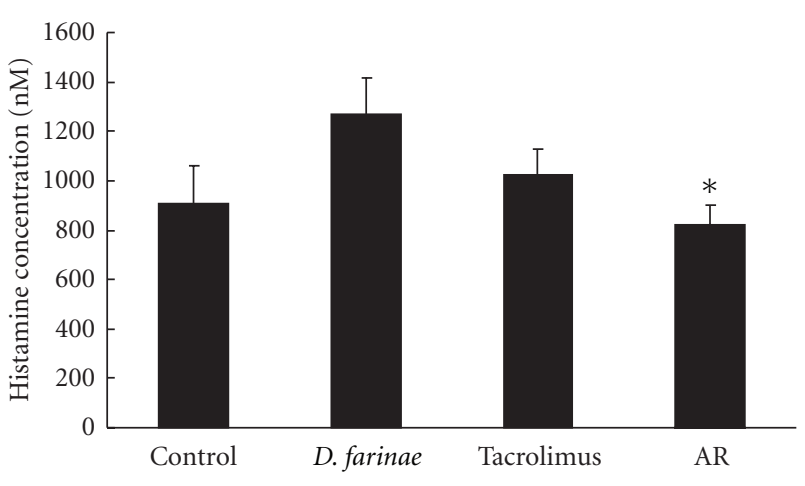

(a)

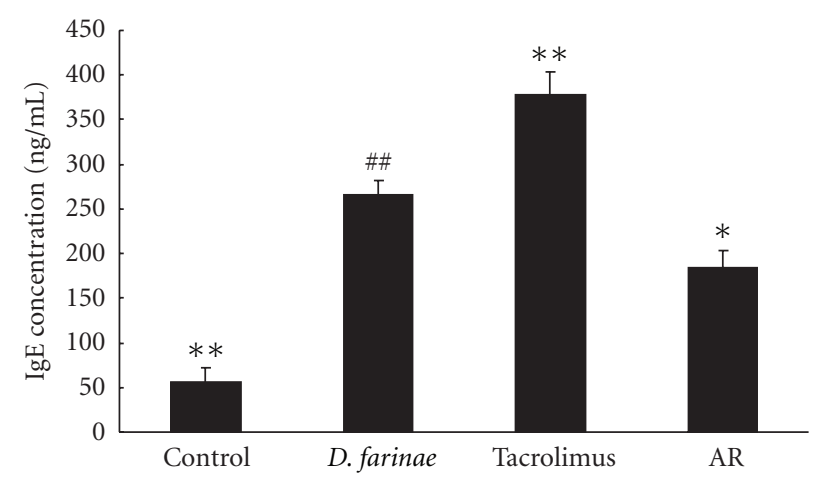

(b)

Figure 2: Effect of Angelicae Dahuricae Radix (AR) on plasma levels of histamine and IgE in Dermatophagoides farinae-induced $\mathrm{AD}$-like lesions in $\mathrm{NC/Nga}$ mice. The groups were untreated (cont), D. farinae plus vehicle-treated (D. farinae), D. farinae plus tacrolimus-treated (tacrolimus), and D. farinae plus AR-treated (AR) NC/Nga mice. The plasma concentrations of histamine (a) and $\operatorname{IgE}(\mathrm{b})$ were measured by ELISA (mean \pm SEM, $n=5$ ), "* $P<0.05$, \#\# $: P<0.01$ compared with control group, ${ }^{*}: P<0.05$, ${ }^{* *}$ : $P<0.01$ compared with $D$. farinae-induced group.

control cells secreted $4.64 \pm 0.54 \mathrm{pg} / \mathrm{mL}$ hTARC. When each cell type was treated with $10 \mathrm{ng} / \mathrm{mL}$ of TNF- $\alpha / \mathrm{IFN}-\gamma$, the hTARC level increased 5.3-fold $(24.72 \pm 3.24 \mathrm{pg} / \mathrm{mL})$. We confirmed that the positive controls induced decreased levels of hTARC in a dose-dependent manner; silymarin-treated cells secreted $9.14 \pm 0.29 \mathrm{pg} / \mathrm{mL}$ at $20 \mu \mathrm{g} / \mathrm{mL}$ and $3.63 \pm$ $0.56 \mathrm{pg} / \mathrm{mL}$ at $50 \mu \mathrm{g} / \mathrm{mL}$, and forskolin-treated cells secreted $13.50 \pm 0.54 \mathrm{pg} / \mathrm{mL}$ at $10 \mu \mathrm{M}$ and $5.72 \pm 1.23 \mathrm{pg} / \mathrm{mL}$ at $30 \mu \mathrm{M}$. AR inhibited TNF- $\alpha / \mathrm{IFN}-\gamma$-induced hTARC production in a dose-dependent manner, corresponding to $26.89 \pm$ $1.00 \mathrm{pg} / \mathrm{mL}, 14.95 \pm 0.56 \mathrm{pg} / \mathrm{mL}$, and $3.41 \pm 1.40 \mathrm{pg} / \mathrm{mL}$ at 60, 150 and $300 \mu \mathrm{g} / \mathrm{mL}$, respectively (Figure 3(b)).

3.5. AR Inhibits the Expression of TARC, MDC, and RANTES. We measured the ability of AR to inhibit transcription of TARC and MDC using RT-PCR. Expression of TARC and MDC was stimulated with TNF- $\alpha / \mathrm{IFN}-\gamma$ treatment of HaCaT. In the positive controls, silymarin and forskolin suppressed the expression of TARC and MDC. AR inhibited 


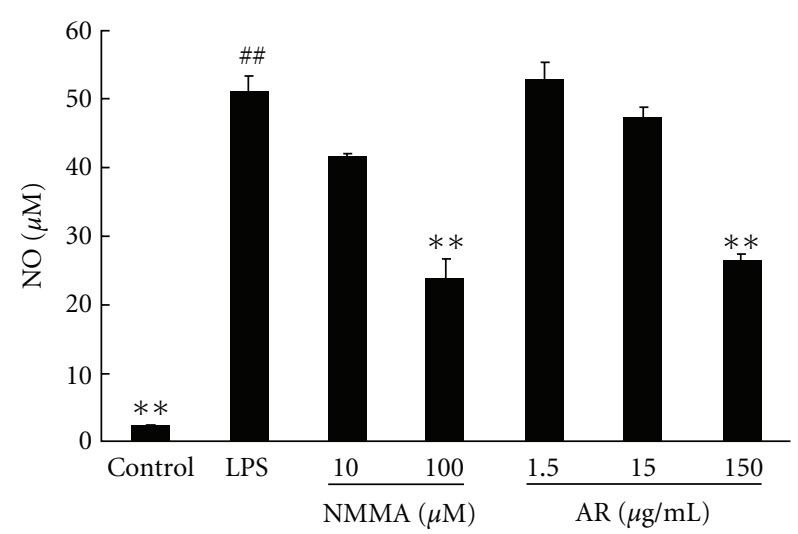

(a)

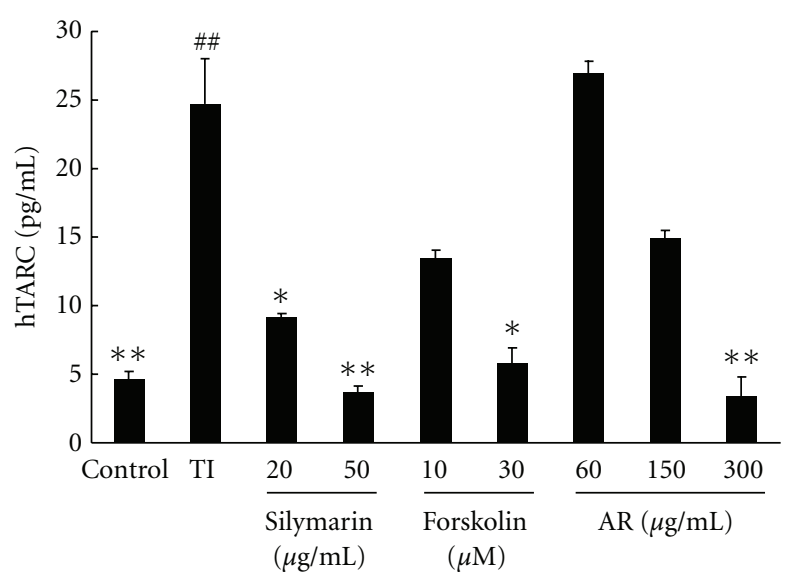

(b)

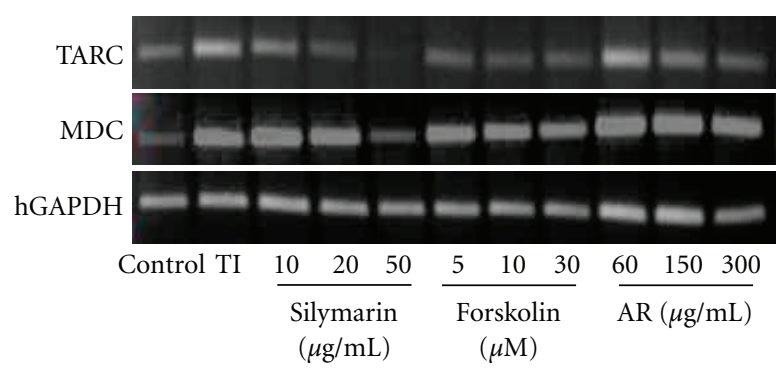

(c)

FIgURe 3: Inhibitory effects on NO and TARC by Angelicae Dahuricae Radix (AR). The concentrations of NO and TARC in the supernatant were determined using NO and TARC ELISA assays. (a) RAW 264.7 cells were treated with LPS ( $1 \mu \mathrm{g} / \mathrm{mL})$ for $18 \mathrm{hr}$. AR inhibited NO production in a concentration-dependent manner (mean $\pm \operatorname{SEM}(n=5),{ }^{\#}: P<0.05,{ }^{\# \#}: P<0.01$ compared with control group, ${ }^{*}: P<0.05$, **: $P<0.01$ compared with the LPS-treated group). (b) HaCaT cells were treated with TNF- $\alpha$ plus IFN- $\gamma$ (TI) $(10 \mathrm{ng} / \mathrm{mL}$ each) for $24 \mathrm{~h}$. TARC levels were significantly suppressed by AR (mean \pm SEM, $n=5$ ), ${ }^{\#}: P<0.05,{ }^{\# \#}: P<0.01$ compared with control group, ${ }^{*}: P<0.05$, ${ }^{* *}: P<0.01$ compared with TI treatment. (c) The MDC and TARC mRNA expression levels were determined by RT-PCR analysis, and AR suppressed the mRNA expression of MDC and TARC. The cells were treated with silymarin (10, 20, or 50 $\mu \mathrm{g} / \mathrm{mL})$, forskolin $(5,10$, or $30 \mu \mathrm{g} / \mathrm{mL})$, or $\operatorname{AR}(60,150$, or $300 \mu \mathrm{g} / \mathrm{mL})$.

the expression of TARC and MDC in a dose-dependent manner (Figure 3(c)).

\section{Discussion}

In this study, we investigated whether AR suppressed the induction of AD-like skin lesions in $D$. farinae-induced $\mathrm{NC} / \mathrm{Nga}$ mice. The NC/Nga mouse model is one of the most valuable mouse models of human AD [17]. This model is used to evaluate drug candidates to treat $\mathrm{AD}$. $\mathrm{AD}$ is an inflammatory disorder that is caused by genetics factors and environmental factors such as dust mites and mold.

Our study confirms that AD-like lesions such as erythema/hemorrhage, edema, excoriation/erosion, and scaling/dryness are observed in the $D$. farinae-induced model. In this model, AR significantly inhibited the development of AD-like skin lesions on both the back and ears of mice. The histological features of the AR-treated group were similar to those of the control group. These results demonstrate that topical AR application has an anti-inflammatory effect on the AD-like skin lesions on NC/Nga mice. AR also suppressed the histamine and IgE levels in plasma to levels comparable to those of the control group. We confirmed that the AR $10 \mathrm{mg} /$ mouse suppressed the AD-like skin lesions, so further experiments carry out application the dose-dependent of AR extracts and fractions.

Immunosuppressive drugs are very effective in the treatment of $\mathrm{AD}$. Two such drugs, tacrolimus and cyclosporine (CsA), bind calcineurin and inhibit nuclear factor of activated T-cell (NF-AT) nuclear translation [18]. Tacrolimus $(0.1 \%, \mathrm{w} / \mathrm{v})$ is used to treat patients with serious $\mathrm{AD}$ over a short period of time, and it suppresses pruritus in AD-like lesions. This treatment also avoids direct application onto inflammation lesions. However, we found that because of adverse effects, this compound is not suitable for use on injuries. In our study, tacrolimus inhibited the increased histamine levels in plasma related with pruritus, whereas the IgE levels were increased by tacrolimus. Hanifin et al. reported that the most common application site adverse events of tacrolimus were pruritus, skin burning (e.g., burning or warmth sensation, stinging), and skin infection (which included all cutaneous infections not otherwise specified, e.g., bacterial infections, molluscum, and pyoderma) [19]. So we changed the positive control to prednisolone in the 
further study (see Figure 1S in Supplementary Material available online at doi:10.1155/2012/743075).

To investigate the anti-inflammatory effect of $\mathrm{AR}$, we estimated NO production in LPS-treated RAW 264.7 cells. It has been shown that physiological levels of NO can play an important role as an immune regulator, neurotransmitter, and a vasodilator in a variety of tissues. The high levels of NO produced by iNOS, however, have been defined as cytotoxic in inflammation and endotoxemia models [20]. Kang et al. reported that ethyl acetate fraction from AR had the antiinflammatory effect [21]. We also confirmed that AR can inhibit NO production in LPS-treated RAW 264.7 cells in a dose-dependent manner.

The pathogenesis of $\mathrm{AD}$ is mediated by $\mathrm{CD} 4^{+} \mathrm{T}$ lymphocytes that produce Th2 cytokines. TARC and MDC function as selective chemoattractants that assist in the recruitment and migration of Th2 cells [22]. TARC and MDC are thought to play important roles in the development of skin diseases such as AD. In this study, we examined the effect of AR extract on hTARC production in TNF- $\alpha /$ IFN- $\gamma$-treated HaCaT cells and on the mRNA expression of TARC and MDC. We used forskolin and silymarin as positive controls to suppress inflammation. In another study, forskolin, a cAMP inhibitor, suppressed TARC and MDC levels in TNF$\alpha /$ IFN- $\gamma$-treated HaCaT cells [23]. Silymarin was also shown to inhibit the $\mathrm{AD}$-like skin lesions in D. pteronyssinusinduced NC/Nga mice and to suppress IL-4 and IgE levels in plasma [24]. In our study, the AR extract suppressed hTARC production in TNF- $\alpha / \mathrm{IFN}-\gamma$-treated HaCaT cells in a dosedependent manner. $\mathrm{AR}$ and immunosuppressive controls were also able to inhibit TARC and MDC mRNA levels. We thought that MDC is less marginal effect than TARC level. AR may therefore regulate the recruitment of Th2type cells to AD lesions by suppressing inflammatory mRNA expression. Some known antiallergic drugs are related to NF$\kappa \mathrm{B}$, STAT1, and p38 activation in activated macrophages and keratinocytes [25]. It will be necessary to assess NF- $\kappa \mathrm{B}$ and p38 in the cells used in our study to determine whether the activity of $\mathrm{AR}$ is associated with an anti-inflammatory or antiallergy effect. AR may, therefore, be an important new anti-inflammatory drug for the treatment of Th2-induced inflammation.

In conclusion, topical AR was shown to be effective in treating $\mathrm{AD}$-like skin lesions in NC/Nga mice and inhibited AD-related gene (TARC and MDC) expression in TNF$\alpha / \mathrm{IFN}-\gamma$-treated HaCaT cells. Topical application of AR may therefore be a novel approach to the treatment of AD.

\section{Acknowledgment}

This research was supported by a Grant from Korea Research Council of Fundamental Science \& Technology (KRCF), no. kiom-2009-s2, Korea.

\section{References}

[1] X. Zheng, X. Zhang, X. Sheng et al., "Simultaneous characterization and quantitation of 11 coumarins in Radix Angelicae Dahuricae by high performance liquid chromatography with electrospray tandem mass spectrometry," Journal of Pharmaceutical and Biomedical Analysis, vol. 51, no. 3, pp. 599-605, 2010.

[2] O. H. Kang, H. S. Chae, Y. C. Oh et al., "Anti-nociceptive and anti-inflammatory effects of angelicae dahuricae radix through inhibition of the expression of inducible nitric oxide synthase and NO production," The American Journal of Chinese Medicine, vol. 36, no. 5, pp. 913-928, 2008.

[3] H. Li, Y. Dai, H. Zhang, and C. Xie, "Pharmacological studies on the Chinese drug radix Angelicae dahuricae," Zhongguo Zhongyao Zazhi, vol. 16, no. 9, pp. 560-576, 1991.

[4] S. Yi, J. Y. Cho, K. S. Lim et al., "Effects of angelicae tenuissima radix, angelicae dahuricae radix and scutellariae radix extracts on cytochrome P450 activities in healthy volunteers," Basic and Clinical Pharmacology and Toxicology, vol. 105, no. 4, pp. 249-256, 2009.

[5] L. You, R. An, X. Wang, and Y. Li, "Discovery of novel osthole derivatives as potential anti-breast cancer treatment," Bioorganic and Medicinal Chemistry Letters, vol. 20, no. 24, pp. 7426-7428, 2010.

[6] H. Nie and Y. J. Shen, "Effect of essential oil of radix angelicae dahuricae on $\beta$-endorphin, ACTH, NO and proopiomelanocortin of pain model rats," Zhongguo Zhongyao Zazhi, vol. 27, no. 9, pp. 690-693, 2002.

[7] Y. Kimura, H. Okuda, and K. Baba, "Histamine-release effectors from Angelica dahurica var. dahurica root," Journal of Natural Products, vol. 60, no. 3, pp. 249-251, 1997.

[8] X. L. Piao, I. H. Park, S. H. Baek, H. Y. Kim, M. K. Park, and J. H. Park, "Antioxidative activity of furanocoumarins isolated from Angelicae dahuricae," Journal of Ethnopharmacology, vol. 93, no. 2-3, pp. 243-246, 2004.

[9] C. A. Akdis, K. Blaser, and M. Akdis, "Apoptosis in tissue inflammation and allergic disease," Current Opinion in Immunology, vol. 16, no. 6, pp. 717-723, 2004.

[10] J. Koo and S. Arain, "Traditional Chinese medicine in dermatology," Clinics in Dermatology, vol. 17, no. 1, pp. 2127, 1999.

[11] K. Jahnz-Rozyk, T. Targowski, E. Paluchowska, W. Owczarek, and A. Kucharczyk, "Serum thymus and activation-regulated chemokine, macrophage-derived chemokine and eotaxin as markers of severity of atopic dermatitis," Allergy, vol. 60, no. 5, pp. 685-688, 2005.

[12] T. Kakinuma, K. Nakamura, M. Wakugawa et al., "Serum macrophage-derived chemokine (MDC) levels are closely related with the disease activity of atopic dermatitis," Clinical and Experimental Immunology, vol. 127, no. 2, pp. 270-273, 2002.

[13] Y. Shimada, K. Takehara, and S. Sato, "Both Th2 and Th1 chemokines (TARC/CCL17, MDC/CCL22, and Mig/CXCL9) are elevated in sera from patients with atopic dermatitis," Journal of Dermatological Science, vol. 34, no. 3, pp. 201-208, 2004.

[14] S. Hashimoto, K. Nakamura, N. Oyama et al., "Macrophagederived chemokine (MDC)/CCL22 produced by monocyte derived dendritic cells reflects the disease activity in patients with atopic dermatitis," Journal of Dermatological Science, vol. 44, no. 2, pp. 93-99, 2006.

[15] M.-Y. Lee, C.-S. Seo, J.-A. Lee et al., "Anti-asthmatic effects of Angelica dahurica against ovalbumin-induced airway inflammation via upregulation of heme oxygenase-1," Food and Chemical Toxicology, vol. 49, no. 4, pp. 829-837, 2011.

[16] M. Hiroshi, W. Naohiro, P. Gregory et al., "Development of atopic dermatitis-like skin lesion with IgE hyperproduction 
in NC/Nga mice," International Immunology, vol. 9, no. 3, pp. 461-466, 1997.

[17] A. Minty, P. Chalon, J. M. Derocq et al., "Interleukin-13 is a new human lymphokine regulating inflammatory and immune responses," Nature, vol. 362, no. 6417, pp. 248-250, 1993.

[18] G. Sandoval-López and L. M. Teran, “TARC: novel mediator of allergic inflammation," Clinical and Experimental Allergy, vol. 31, no. 12, pp. 1809-1812, 2001.

[19] J. M. Hanifin, A. S. Paller, L. Eichenfield et al., "Efficacy and safety of tacrolimus ointment treatment for up to 4 years in patients with atopic dermatitis," Journal of the American Academy of Dermatology, vol. 53, no. 2, pp. S186-S194, 2005.

[20] S. Moncada, R. M. J. Palmer, and E. A. Higgs, "Nitric oxide: physiology, pathophysiology, and pharmacology," Pharmacological Reviews, vol. 43, no. 2, pp. 109-142, 1991.

[21] O. H. Kang, G. H. Lee, H. J. Choi et al., "Ethyl acetate extract from Angelica Dahuricae Radix inhibits lipopolysaccharideinduced production of nitric oxide, prostaglandin E2 and tumor necrosis factor- $\alpha$ via mitogen-activated protein kinases and nuclear factor- $\kappa \mathrm{B}$ in macrophages," Pharmacological Research, vol. 55, no. 4, pp. 263-270, 2007.

[22] N. Takashi, H. Kunio, N. Daisuke et al., "Selective induction of Th2-attracting chemokines CCL17 and CCL22 in human B cells by latent membrane protein 1 of epstein-barr virus," Journal of Virology, vol. 78, no. 4, pp. 1665-1674, 2004.

[23] X. F. Qi, D. H. Kim, Y. S. Yoon et al., "The adenylyl cyclasecAMP system suppresses TARC/CCL17 and MDC/CCL22 production through p38 MAPK and NF- $\kappa$ B in HaCaT keratinocytes," Molecular Immunology, vol. 46, no. 10, pp. 19251934, 2009.

[24] J. S. Kang, W. K. Yoon, M. H. Han et al., "Inhibition of atopic dermatitis by topical application of silymarin in NC/Nga mice," International Immunopharmacology, vol. 8, no. 10, pp. 1475-1480, 2008.

[25] M. Hämäläinen, R. Nieminen, P. Vuorela, M. Heinonen, and E. Moilanen, "Anti-inflammatory effects of flavonoids: genistein, kaempferol, quercetin, and daidzein inhibit STAT1 and NF- $\kappa$ B activations, whereas flavone, isorhamnetin, naringenin, and pelargonidin inhibit only NF- $\kappa \mathrm{B}$ activation along with their inhibitory effect on iNOS expression and NO production in activated macrophages," Mediators of Inflammation, vol. 2007, Article ID 45673, 10 pages, 2007. 


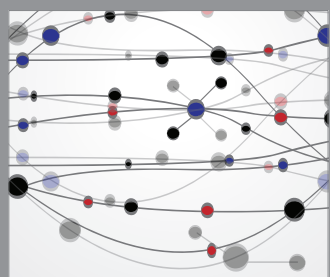

The Scientific World Journal
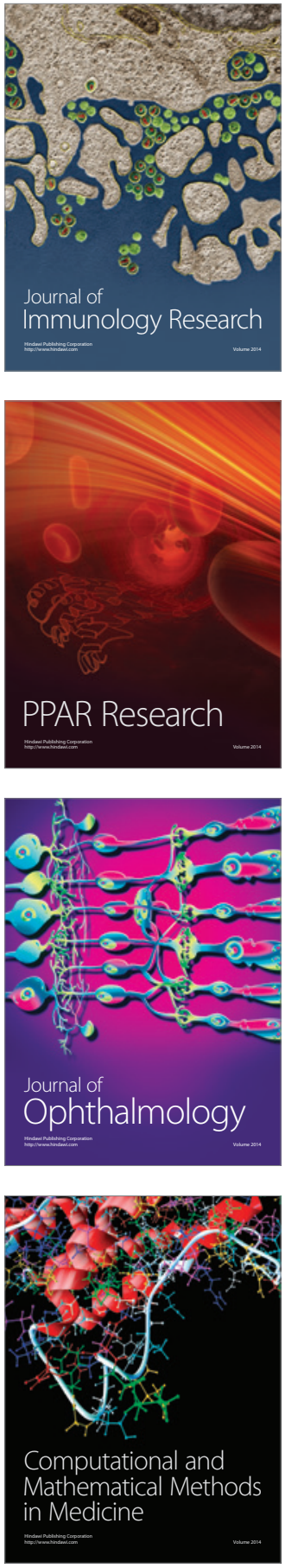

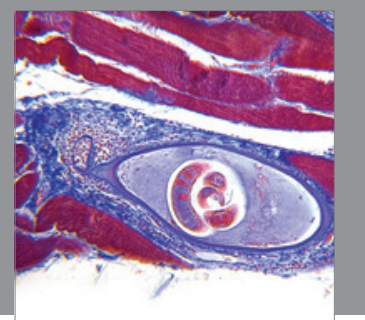

Gastroenterology

Research and Practice
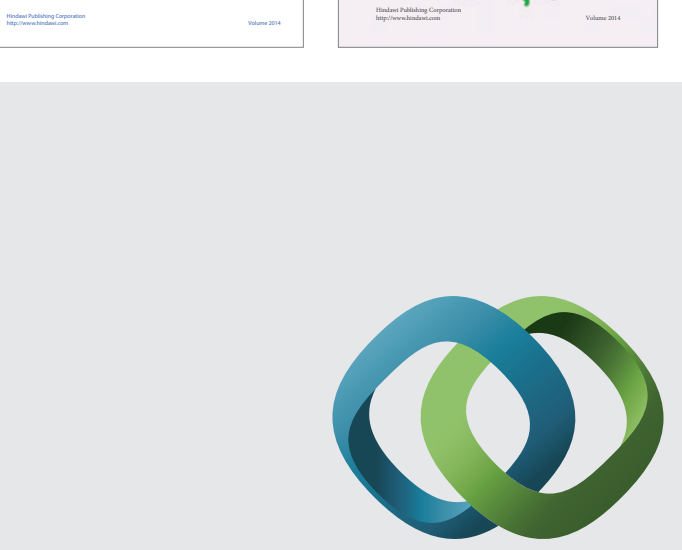

\section{Hindawi}

Submit your manuscripts at

http://www.hindawi.com
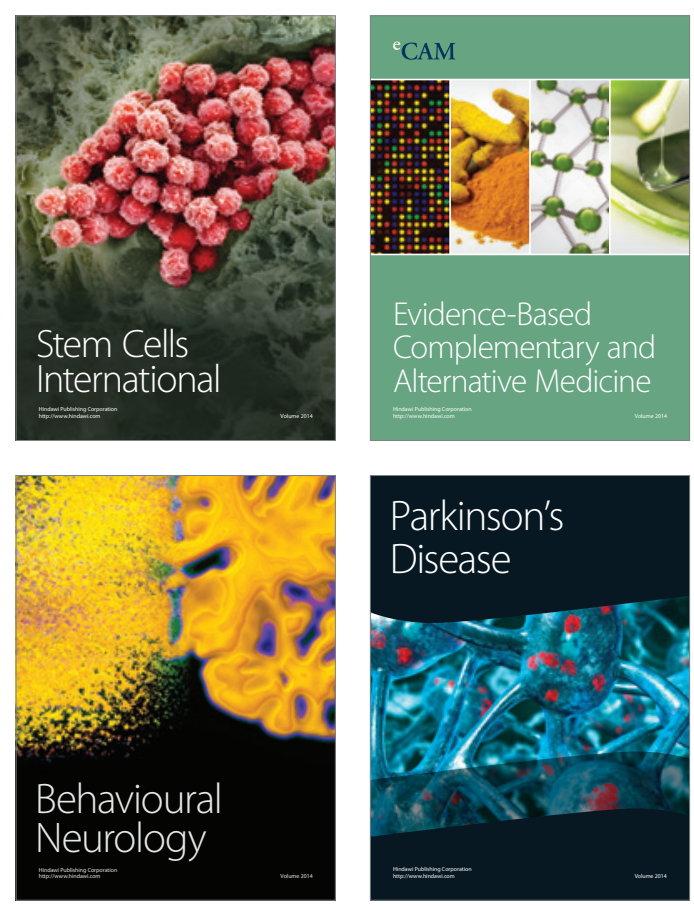

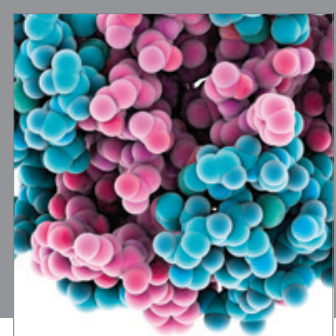

Journal of
Diabetes Research

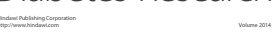

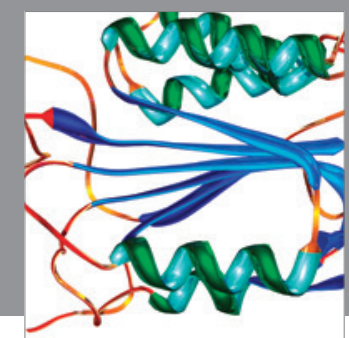

Disease Markers
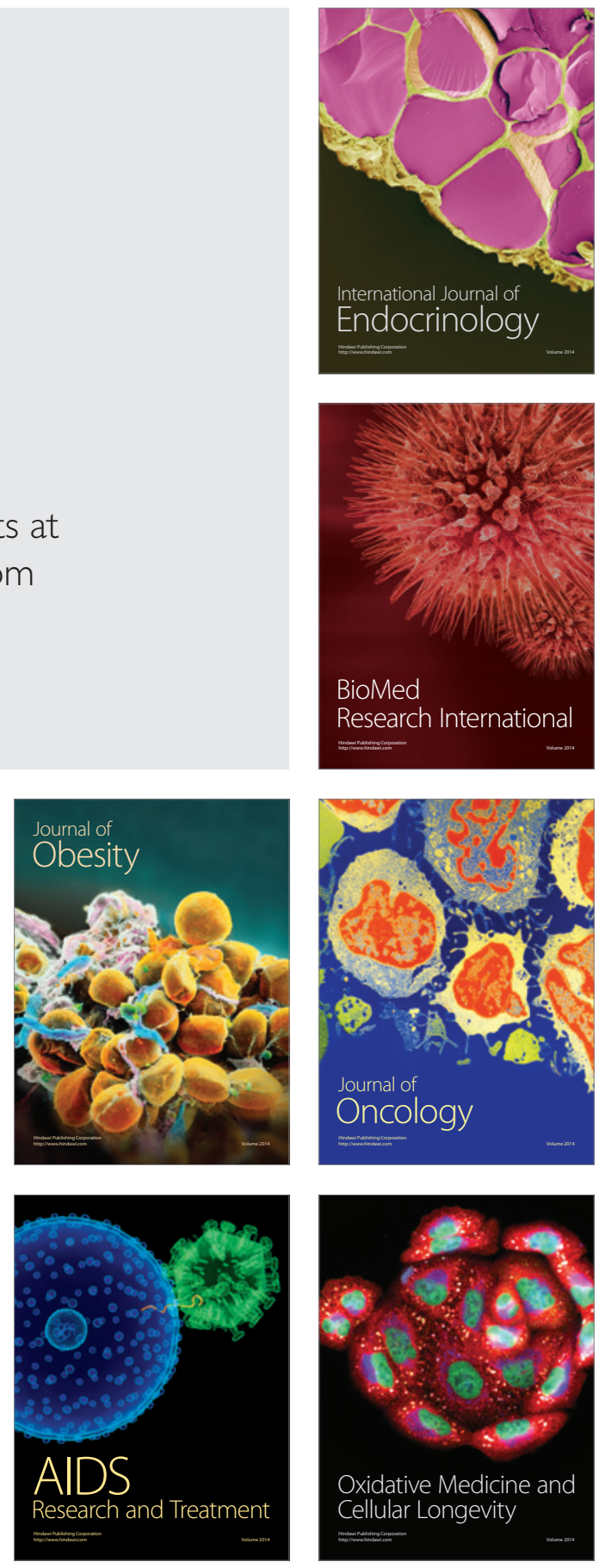